\title{
Fuzzy Reasoning based on DNA Computing
}

\author{
Kumar S. Ray \\ Indian Statistical Institute \\ 203, B. T. Road \\ Kolkata, India
}

\author{
Piyali Chatterjee \\ Indian Statistical Institute \\ 203, B. T. Road \\ Kolkata, India.
}

\begin{abstract}
Fuzzy reasoning based on fuzzy logic was first proposed by Zadeh to handle the vague concept of reasoning in our day to day life. Since the inception of fuzzy reasoning several extensions, specifications and applications on fuzzy reasoning have been developed by different researchers. Fuzzy reasoning is now a very well established method and has a tremendous potentiality in different application areas of science and engineering. In this paper we realize fuzzy reasoning by DNA computing with an aim to add a new dimension to the existing fuzzy reasoning method by bringing it down to nanoscale computing. We replace the logical aspect of fuzzy reasoning by DNA chemistry. To achieve this goal we first fuzzify the synthetic DNA sequence by quantum dot which is a recent advancement of nanotechnology. Thus with the help of fuzzy DNA, which holds the vague concept of human reasoning, we realize the basic method of fuzzy reasoning on a DNA chip. In this approach we can avoid the tedious choice of a suitable implication operator (for a particular application) necessary for existing fuzzy reasoning based on fuzzy logic. The inferred consequences obtained from DNA computing base fuzzy reasoning is ultimately hybridized with appropriate complementary sequence probed on a DNA chip to confirm the result of inference.
\end{abstract}

The full text of the article is not available in the cache. Kindly refer the IJCA digital library at www.ijcaonline.org for the complete article. In case, you face problems while downloading the full-text, please send a mail to editor at editor@ijcaonline.org 